\title{
Interlayer coupling in peroxitonic model of superconductivity
}

\author{
R RAI \\ National Physical Laboratory, New Delhi 110012, India
}

\begin{abstract}
The unusual ESR spectra of $\mathrm{YBa}_{2} \mathrm{Cu}_{3} \mathrm{O}_{7-x}$ is ascribed to a $\mathrm{Cu}^{2+}$ ion, whose one ligand in $\mathrm{CuO}_{2}$ plane is involved in a peroxiton $\mathrm{O}^{-}-\mathrm{Cu}^{+}-\mathrm{O}^{-}$bond. The analysis shows that the presence of a ligand in $\mathrm{O}^{-}$substantially increases the energies of $d$-hole orbitals. Since this increase for $d_{x^{2}-v^{2}}$ orbital is much larger than that for $d_{3 z^{2}-r^{2}}$ orbital, their separation is drastically reduced and there occurs a significant orthorhombic mixing between the two. This charge transfer from a planar $d_{x^{2}-\gamma^{2}}$ orbital to a $d_{3 z^{2}-r^{2}}$ orbital, which lies mainly perpendicular to the plane, provides a mechanism for interlayer coupling. The effect of this coupling on superconducting transition temperature is discussed.
\end{abstract}

Keywords. Interlayer coupling; peroxitonic model.

\section{Introduction}

Nearly isotropic ESR spectrum of $\mathrm{YBaCu}_{3} \mathrm{O}_{7-x}$ observed by Meheran et al (1988) was recently explained (Rai 1989) by ascribing it to a $\mathrm{Cu}^{2+}$ ion, whose one ligand in $\mathrm{CuO}_{2}$ plane is involved in a $\mathrm{O}^{-}-\mathrm{Cu}^{+}-\mathrm{O}^{-}$peroxiton bond with an oxygen and copper of neighbouring $\mathrm{CuO}_{2}$ unit (figure 1). The analysis shows that the presence of a ligand in $\mathrm{O}^{-}$form, instead of $\mathrm{O}^{2-}$, drastically reduces the separation between $d_{x^{2}-y^{2}}$ and $d_{3 z^{2}-r^{2}}$ orbitals and causes a significant orthorhombic mixing between the two. Thus there occurs an oxygen hole-assisted charge transfer from a planar $d_{x^{2}-y^{2}}$ orbital to a $d_{3 z^{2}-r^{2}}$ orbital, which lies mainly in a direction perpendicular to the plane. A simultaneous charge transfer of this type at the nearest copper sites on two adjacent $\mathrm{CuO}_{2}$ planes will affect an out-of-phase displacement of bridge $\mathrm{O}_{4}$ oxygen atoms in the direction perpendicular to the planes (figure 1). This provides a mechanism for interlayer coupling. Our aim in the present paper is to investigate the effect of this coupling on superconducting transition temperature.

\section{Theory}

The appropriate hamiltonian can be written as

$$
\begin{aligned}
H= & H_{0}+H^{\prime}, \\
H_{0}= & \sum_{K \sigma}\left[\left(t_{k}^{\varepsilon}+E_{\varepsilon}\right) n_{k \sigma}^{\varepsilon}+t_{k}^{\pi} \pi_{k \sigma}^{+} \pi_{k \sigma}\right]+\sum_{\substack{\langle\alpha\rangle\langle i j\rangle \\
\sigma \sigma^{\prime}}}\left[u \sum_{\substack{\left\langle r^{\prime}\right) \\
=(\varepsilon \theta)}} n_{\alpha \sigma}^{r} n_{\alpha \sigma^{\prime}}^{r^{\prime}}\right. \\
& \left.+U_{\pi} n_{i \uparrow}^{\pi} n_{i \downarrow}^{\pi}-J \pi_{i \uparrow}^{+} \pi_{j !}^{+} \pi_{j \uparrow} \pi_{i \downarrow}+h\left(\varepsilon_{\alpha \sigma}^{+} \pi_{i \sigma}+\pi_{i \sigma}^{+} \varepsilon_{\alpha \sigma}\right)+Q_{i j} n_{i \sigma}^{\pi} n_{j-\sigma}^{\pi}\right], \\
H^{\prime}= & \sum_{\substack{\langle i \alpha\rangle\left\langle\alpha \alpha^{\prime}\right\rangle \perp \\
\sigma}}\left[E_{\theta} \theta_{\alpha \sigma}^{+} \theta_{\alpha \sigma}+\left(V_{\varepsilon} n_{\alpha \sigma}^{\varepsilon}+V_{\theta} n_{\alpha \sigma}^{\varepsilon}\right) n_{i \sigma^{\prime}}^{\pi}+d_{i} n_{i \sigma^{\prime}}^{\pi}\left(\varepsilon_{\alpha \sigma}^{+} \theta_{\alpha \sigma}+\theta_{\alpha \sigma}^{+} \varepsilon_{\alpha \sigma}\right)\right. \\
& \left.+\left\{\hbar w b_{\alpha \alpha \alpha^{\prime}}^{+} b_{\alpha \alpha^{\prime}}+\lambda_{\perp}\left(\frac{\hbar}{2 \mu_{\perp} w_{\perp}}\right)^{1 / 2}\left(b_{\alpha \alpha^{\prime}}^{+}+b_{\alpha \alpha^{\prime}}\right)\right\} n_{\alpha \sigma}^{\theta} n_{\alpha \sigma^{\prime} \sigma^{\prime}}^{\theta}\right] .
\end{aligned}
$$



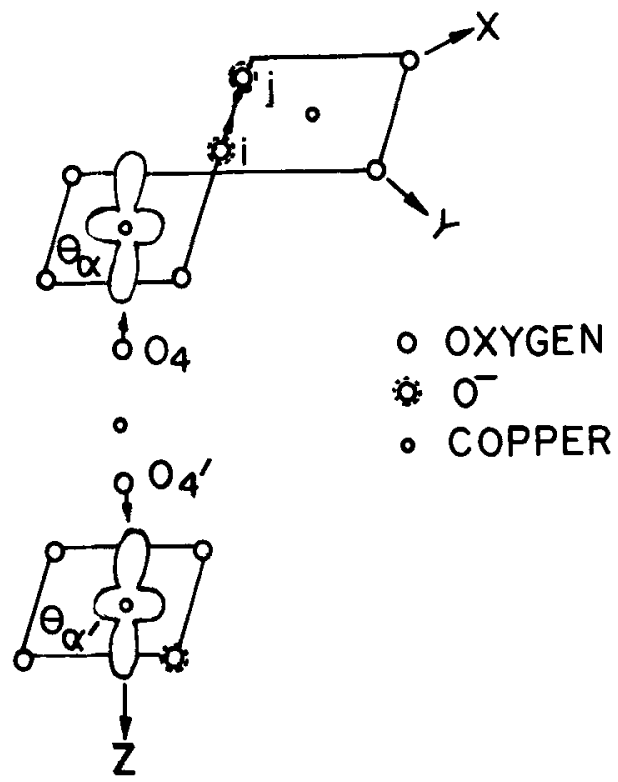

- COPPER

Figure 1. Occupancy of $d_{3 z^{2}-r^{2}}$ orbital affected by a $\mathrm{O}^{-}$ligand and an out-of-these displacement of bridge $\mathrm{O}_{4}$ and $\mathrm{O}_{4}$, oxygen atoms due to simultaneous occupancy of $d_{3 z^{2}-r^{2}}$ orbitals on sites $\alpha$ and $\alpha^{\prime}$, the nearest neighbours on two adjacent $\mathrm{CuO}_{2}$ planes.

$H_{0}$ is essentially the hamiltonian proposed by Chakraverty et al (1987). Here E refers to the hybridized $d_{x^{2}-y^{2}}-p_{\sigma}$ antibonding orbital, $\theta$ to $s_{3 z^{2}-r^{2}}$ and $\pi$ to oxygen $p_{\pi}$. The operators $\varepsilon^{+}(\varepsilon)$ etc are the corresponding band or site hole creation (annihilation) operators. $Q_{i j}$ involves the $O_{i}-O_{j}$ bond fluctuation in a $\mathrm{CuO}_{2}$ plane due to the presence or absence of a hole on each of these two oxygen atoms.

The quantities $V_{\varepsilon}$ and $V_{\theta}$ in $H^{\prime}$ are respectively the increase in Coulomb energies of $\varepsilon$ and $\theta$ hole orbitals due to the presence of a hole on a nearest neighbour oxygen site in $\mathrm{CuO}_{2}$ plane, $d_{i}=d$ or $-d$ depending upon whether the site $i$ is on $x$ or $y$ axis (figure 1) and $d$ is an orthorhombic parameter (Rai 1989). The notation $\left\langle\alpha \alpha^{\prime}\right\rangle_{\perp}$ indicates that $\alpha$ and $\alpha^{\prime}$ are the nearest copper sites on two adjacent $\mathrm{CuO}_{2}$ planes. The presence or absence of a hole on each of the two $\theta_{\alpha}$ and $\theta_{\alpha^{\prime}}$ orbitals is responsible for the out-of-phase oscillation of the two bridge $\mathrm{O}_{4}$ oxygen atoms (figure 1) and $b_{\alpha x^{\prime}}^{+}\left(b_{\alpha \alpha^{\prime}}\right)$ are the phonon creation (destruction) operators corresponding to this oscillation.

Using a unitary transformation

$$
\bar{H}=u^{+} H u
$$

with

$$
u=\prod_{\langle\alpha i\rangle \sigma \sigma^{\prime}} \exp \left[\Phi_{i}\left(\varepsilon_{\alpha \sigma}^{+} \theta_{\alpha \sigma}-\theta_{\alpha \sigma}^{+} \varepsilon_{\alpha \sigma}\right) n_{i \sigma^{\prime}}^{\pi}\right.
$$

and

$$
\left.\tan 2 \Phi_{i}=2 d_{i} / E_{\theta}+V_{\theta}-E_{\varepsilon}-V_{\varepsilon}\right] .
$$

Neglecting the interactions between three or more sites in the same $\mathrm{CuO}_{2}$ plane we 
notice that, excepting the terms with $h$ and $\lambda_{i}$ as coefficients, the mixing between $\varepsilon$ and $\theta$ orbitals is eliminated. The $h$ and $\lambda_{i}$ dependent terms are modified as

$$
\sum_{\langle x\rangle \sigma \sigma} h\left[1+\left(1-\cos \Phi_{i}\right) n_{\alpha \sigma}^{\theta}\right]\left(\varepsilon_{\alpha \sigma}^{+} \theta_{\alpha \sigma}+\theta_{\alpha \sigma}^{+} \varepsilon_{\alpha \sigma}\right)
$$

and

$$
\begin{aligned}
& \sum_{\substack{\langle\alpha\rangle_{\perp}\left\langle i \alpha^{\prime}\right\rangle \\
\sigma \sigma \sigma^{\prime}}} \lambda_{\perp}\left(\frac{\hbar}{2 \mu_{\perp} w_{\perp}}\right)^{1 / 2}\left(b_{\alpha \sigma^{\prime}}^{+}+b_{\alpha \alpha^{\prime}}\right) n_{\alpha^{\prime} \sigma^{\prime}}^{\theta}\left\{n_{\alpha \sigma}^{\varepsilon} n_{i \sigma^{\prime}}^{\pi} \sin ^{2} \Phi_{i}\right. \\
& \left.+n_{\alpha \sigma}^{\theta} \cos ^{2} \Phi_{i}-\frac{1}{2} \sin 2 \Phi_{i}\left(\varepsilon_{\alpha \sigma}^{+} \theta_{\alpha \sigma}+\theta_{\alpha \sigma^{\prime}}^{+} \varepsilon_{\alpha \sigma}\right) n_{i \sigma^{\prime}}^{\pi}\right\}
\end{aligned}
$$

Neglecting the last term in the curly bracket in (8), dropping all terms in $\bar{H}$ which involve only $\theta$-dependence, and using the Lange-Firsov transformation operator

$$
S=\exp \left[-\sum_{\substack{\left\langle\alpha \alpha^{\prime}\right\rangle\langle\alpha i\rangle \\ \sigma \sigma^{\prime}}} \lambda_{\perp}\left(\frac{\hbar}{2 \mu_{\perp} w_{\perp}}\right)^{1 / 2} \frac{1}{h w_{\perp}}\left(b_{\alpha \alpha^{\prime}}^{+}-b_{\alpha \alpha^{\prime}}\right) n_{\alpha^{\prime} \sigma^{\prime}}^{\theta} n_{i \sigma^{\prime}}^{\pi} n_{\alpha \sigma}^{\varepsilon} \sin ^{2} \Phi_{i}\right]
$$

it is seen that the zero phonon expectation value of $\left(S^{+} \bar{H} S\right)$ regains the form of the original hamiltonian (Chakraverty et al 1987 ) with renormalized functions $E_{\varepsilon}$ and $h$ given by

$$
\begin{aligned}
& E_{\varepsilon}^{\prime}=E_{\varepsilon}+\frac{\delta}{2}\left\{E_{\theta}+V_{\theta}+V_{\varepsilon}-E_{\varepsilon}-\left[4 d^{2}+\left(E_{\theta}+V_{\theta}-E_{\varepsilon}-V_{\varepsilon}\right)^{2}\right]^{1 / 2}\right\} \\
& h^{\prime}=h\left[1+a_{1} \delta+a_{2} \delta^{2}+a_{3} \delta^{3}\right] \\
& \delta=(1-2 x)=\left\langle n_{i \sigma}^{\pi}\right\rangle=\frac{1}{2 \sin ^{2} \Phi}\left\langle n_{\alpha \sigma}^{\theta}\right\rangle \\
& a_{1}=2 \sin ^{2} \Phi(1-\cos \Phi) \\
& a_{2}=2 q_{-} \sin ^{2} \Phi\left(1-4 \sin ^{2} \Phi-2 \sin ^{2} \Phi \cos \Phi\right) \\
& a_{3}=4 q_{+} \sin ^{4} \Phi\left(4-2 \cos ^{2} \Phi\right)+4 q_{-} \sin ^{4} \Phi(1-\cos \Phi), \\
& q_{-}=\exp \left(-\frac{2 \sin ^{4} \Phi}{2 \hbar \mu_{\perp} w_{\perp} 3}\right)-1 \\
& q_{+}=\exp \left(\frac{\lambda_{\perp}^{2} \sin ^{4} \Phi}{2 \hbar \mu_{\perp} w_{\perp} 3}\right)-1
\end{aligned}
$$

In writing (12) we have noted that the expectation value of a hole at any oxygen site in a $\mathrm{CuO}_{2}$ plane is $\frac{1}{2}(1-2 x)$. The expectation value of $n_{\alpha a}^{\theta}$ is non-zero only if one ligand of $\mathrm{Cu}^{2+}$ ion at $\alpha$ is in $\mathrm{O}^{-}$form so that there is an orthorhombic mixing between $\left|\varepsilon_{\alpha \sigma}\right\rangle$ and $\left|\theta_{\alpha \sigma}\right\rangle$ orbitals resulting in a combined orbital of the form $\left(\cos \Phi\left|\varepsilon_{\alpha \sigma}\right\rangle \pm \sin \Phi \mid \theta_{\alpha \sigma}\right)$ (Rai 1989 ). Since there are two oxygen sites per copper in a $\mathrm{CuO}_{2}$ unit $\left\langle n_{\alpha \sigma}^{\theta}\right\rangle=\sin ^{2} \Phi$ $(1-2 x)$.

Noting that the superconducting transition temperature in a peroxiton model is proportional to $h^{2}$ a relation between $T_{c}$ and the doping parameter $\delta$ can readily be written as

$$
T_{c}=T_{0}\left[1+a_{1} \delta+a_{2} \dot{\delta}^{2}+a_{3} \delta^{3}\right]^{2}
$$




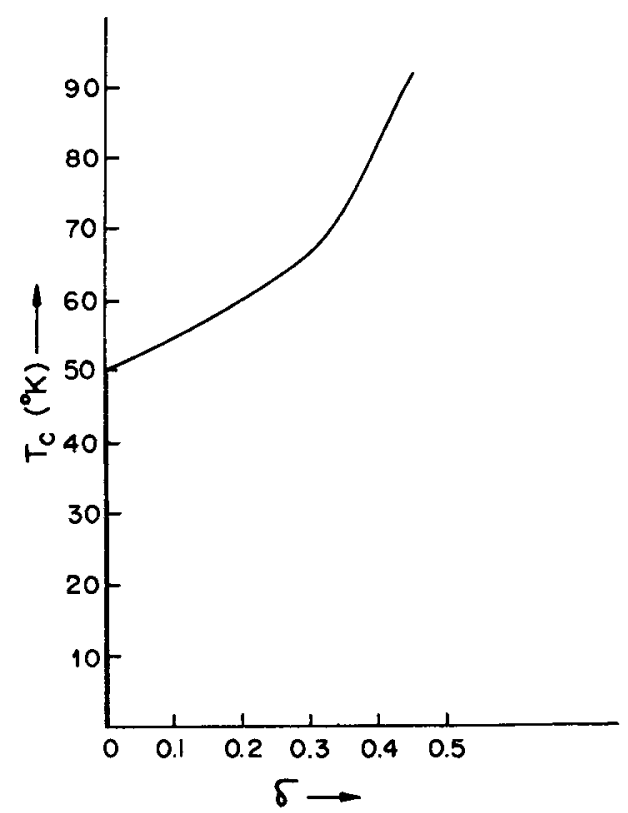

Figure 2. Variation of superconducting transition temperature $T_{c}$ versus doping parameter $\delta=0.5-x$ for $\mathrm{YBa}_{2} \mathrm{Cu}_{3} \mathrm{O}_{7-x}$.

\section{Discussion}

Figure 2 shows a plot of $T_{c}$ versus $\delta$ for the value of $T_{0}=50^{\circ} \mathrm{K},\left(\lambda_{\perp} /\left(2 h \mu W_{\perp}^{3}\right)^{1 / 2}=1 \cdot 208\right.$ and $\Phi=55 \cdot 2^{\circ}$. These parameter values are chosen to simulate the experimental $T_{c}$ versus $\delta$ curve (Goucharov et al 1988) as best as possible. Even though the curve near $\delta \approx 0.2$ does not resemble the experimental $60^{\circ} \mathrm{K}$ plateau the slope in this region is much slower than that above $\delta=0 \cdot 3$. Above $\delta=0 \cdot 3$ the present plot resembles the experimental curve (figure 2). The present value of $\Phi=55.2^{\circ}$ is close to the value of $\Phi=49^{\circ}$ obtained after a phase correction of the value required to explain the ESR result (Rai 1989). The present calculation clearly brings out the importance of oscillation of $\mathrm{O}_{4}$ bridge oxygen atoms in interlayer coupling. The importance of these oscillations is also emphasized in experimental infrared and Raman spectroscopic results (Goncharo et al 1988; Yonggang et al 1988).

\section{Acknowledgement}

The author is grateful to Dr S K Joshi for encouragement.

\section{References}

Chakraverty B K, Feinberg D, Hang Z and Avignon M 1987 Solid State Commun. 641147

Goucharov A F, Denisov V N, Zibrou I P, Marvin B N, Podobedov V B, Sharpro Y Ya and Stishov S M 1988 JETP Lett. 48497

Meheran F, Barnes S E, McGuire T R, Dinger T R, Kaiser D L and Holtzberg F 1988 Solid State Commun. 66299

Rai R 1989 Physica C160259

Yonggang Z, Leiming X, Chen I, Tiangsheng S, Le W and Peng I 1988 Solid State Commun. 68847 